\title{
Limits of noise and confusion in the MWA GLEAM year 1 survey
}

Thomas M. O. Franzen ${ }^{*}{ }^{1}$ Carole A. Jackson, ${ }^{1}$ Joseph R. Callingham, $, 3,2,4$ Ron D. Ekers, ${ }^{1,2}$ Paul J. Hancock, ${ }^{1,2}$ Natasha Hurley-Walker, ${ }^{1}$ John Morgan, ${ }^{1}$ Nick Seymour, ${ }^{1}$ Randall B. Wayth, ${ }^{1,2}$ Sarah V. White, ${ }^{1}$ Martin E. Bell, ${ }^{4,2}$ K. S. Dwarakanath, ${ }^{5}$ Bi-Qing For, ${ }^{6}$ Bryan M. Gaensler, ${ }^{7,2}$ Luke Hindson, ${ }^{8}$ Melanie Johnston-Hollitt, ${ }^{8}$ Anna D. Kapinska, ${ }^{6,2}$ Emil Lenc, ${ }^{3,2}$ Ben McKinley, ${ }^{9,2}$ André R. Offringa, ${ }^{10}$ Pietro Procopio, ${ }^{9,2}$ Lister Staveley-Smith, ${ }^{6,2}$ Chen Wu, ${ }^{6}$ and Qian Zheng ${ }^{8}$

${ }^{1}$ ICRAR Curtin University, Australia; ${ }^{2}$ ARC Centre of Excellence for All-sky Astrophysics (CAASTRO); ${ }^{3}$ University of Sydney, Australia $;{ }^{4}$ CSIRO Astronomy and Space Science (CASS), Australia; ${ }^{5}$ Raman Research Institute, India; ${ }^{6}$ ICRAR University of Western Australia, Australia: ${ }^{7}$ Dunlap Institute for Astronomy \& Astrophysics, University of Toronto, Canada $;{ }^{8}$ Victoria University of Wellington, New Zealand; ${ }^{9}$ The University of Melbourne, Australia, ${ }^{10}$ Netherlands Institute for Radio Astronomy (ASTRON), The Netherlands E-mail: thomas.franzen@curtin.edu.au

The GaLactic and Extragalactic All-sky MWA survey (GLEAM) is a new relatively low resolution, contiguous $72-231 \mathrm{MHz}$ survey of the entire sky south of declination $+25^{\circ}$. In this paper, we outline one approach to determine the relative contribution of system noise, classical confusion and sidelobe confusion in GLEAM images. An understanding of the noise and confusion properties of GLEAM is essential if we are to fully exploit GLEAM data and improve the design of future low-frequency surveys. Our early results indicate that sidelobe confusion dominates over the entire frequency range, implying that enhancements in data processing have the potential to further reduce the noise.

EXTRA-RADSUR2015 (*)

20-23 October 2015

Bologna, Italy

(*) This conference has been organized with the support of the Ministry of Foreign Affairs and International Cooperation, Directorate General for the Country Promotion (Bilateral Grant Agreement ZA14GR02 - Mapping the Universe on the Pathway to SKA)

\footnotetext{
* Speaker.
} 


\section{Introduction}

The Galactic and Extragalactic All-sky MWA survey (GLEAM; Wayth et al., 2015), conducted with the Murchison Widefield Array (MWA; Tingay et al., 2013), covers the declination range $-80^{\circ}$ to $+25^{\circ}$ at $72-231 \mathrm{MHz}$. GLEAM observing began in August 2013 and its primary output from the first year of observations is a catalogue of approximately 300,000 extragalactic radio components (Hurley-Walker et al., in preparation).

Large-area $\left(>100 \mathrm{deg}^{2}\right)$ surveys at low frequencies $(\lesssim 200 \mathrm{MHz})$ such as GLEAM are limited by confusion effects at the mJy level, mainly due to large instrumental beam sizes. The situation is expected to improve with the extensive baselines and sensitivity of the Low Frequency Array (LOFAR; van Haarlem et al., 2013) and Square Kilometre Array Low (Dewdney et al., 2012), which should push this limit substantially fainter.

There are three basic sources of error in a low-frequency image formed with an array: the system noise, classical confusion and sidelobe confusion, where we take sidelobe confusion to include calibration errors. In this paper, we analyse the relative contribution of system noise, classical confusion and sidelobe confusion as a function of frequency in one of the deepest regions of GLEAM. An understanding of the noise and confusion properties is essential if we are to fully exploit GLEAM for extragalactic radio source studies. This is also important for assessing whether enhancements in the data processing, such as improved deconvolution techniques, have the potential to further reduce the noise.

\section{MWA GLEAM observations and imaging strategy}

The MWA consists of 128 16-dipole antenna 'tiles' distributed over an area approximately $3 \mathrm{~km}$ in diameter. It operates at frequencies between 72 and $300 \mathrm{MHz}$, with an instantaneous bandwidth of $30.72 \mathrm{MHz}$. Given the effective width $(\approx 4 \mathrm{~m})$ of the MWA's antenna tiles, the primary beam FWHM at $154 \mathrm{MHz}$ is $27 \mathrm{deg}$. The angular resolution, using a uniform image weighting scheme, is approximately $2.5 \times 2.2 \sec \left(\delta+26.7^{\circ}\right) \operatorname{arcmin}$. The excellent snapshot $u v$ coverage of the MWA and its huge field-of-view (FoV) allow it to rapidly image large areas of sky.

The survey strategy is outlined in Wayth et al. (2015) while the data reduction and analysis methods for the GLEAM year 1 catalogue will be described in detail in Hurley-Walker et al., in preparation. Briefly, meridian drift scans were used to cover the entire sky visible to the MWA. The sky was divided into seven declination strips and five $30.72 \mathrm{MHz}$ bands. The observations were conducted as a series of 2-min scans for each frequency, cycling through all five frequency settings over $10 \mathrm{~min}$. The 2-min snapshots were divided into four $7.68 \mathrm{MHz}$ subbands and imaged separately with robust $=-1$ weighting using WSCLEAN (Offringa et al., 2014). The snapshots for each subband were corrected for the primary beam and mosaicked together.

A deep wideband image covering $170-231 \mathrm{MHz}$ was formed by combining the eight highest frequency subband images. This wideband image was used for source detection and flux density estimates were performed across the 20 7.68-MHz subbands. This approach maximised the number of sources catalogued and provided measurements for them across the full frequency range. 


\section{Noise contribution}

In Section 3.1, we follow Wayth et al. (2015) to estimate the system noise in the GLEAM 7.68 MHz subband mosaics in a 'cold' region of extragalactic sky near zenith. Observations with the Giant Metrewave Radio Telescope by Intema et al. (2011), Ghosh et al. (2012) and Williams, Intema \& Röttgering (2013) probe the $153 \mathrm{MHz}$ counts down to 6, 12 and $15 \mathrm{mJy}$, respectively. In Section 3.2, we use these deep source counts to estimate the classical confusion noise across the GLEAM frequency range. Finally, in Section 3.3, we compare our estimates of the system noise and classical confusion noise with the measured rms noise to draw conclusions about the degree of sidelobe confusion.

\subsection{System noise}

The system noise, $T_{\mathrm{sys}}$, is the Gaussian random noise resulting from the noise power entering the system and is equal to $T_{\text {sky }}+T_{\text {rec }}$, where $T_{\text {sky }}$ is the sky noise and $T_{\text {rec }}$ the receiver noise. For the MWA, $T_{\text {sys }}$ is dominated by $T_{\text {sky }}$ over the entire frequency range covered by GLEAM, with a much smaller contribution from $T_{\text {rec }}(\approx 50 \mathrm{~K})$. It follows that $T_{\text {sys }}$ strongly depends on the region of sky being observed.

The expected beam-weighted average sky temperature over the range of frequencies, pointings and LSTs relevant to GLEAM was calculated by Wayth et al. (2015). Fig. 1 shows the beamweighted average sky temperature at the central frequency of each $30.72 \mathrm{MHz}$ band in a 'cold' region of extragalactic sky. We fit a power law to these data points, obtaining a slope of -2.42 .

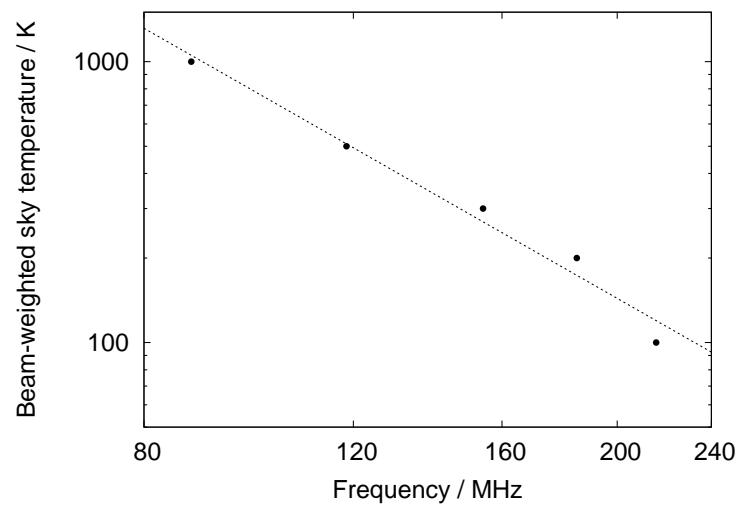

Figure 1: Beam-weighted sky temperature as a function of frequency in a 'cold' region of extragalactic sky at $\delta=-26.7 \mathrm{deg}$ and LST $=0 \mathrm{~h}$. The dashed line is a power-law fit to the data points.

From noise-only simulations that match the GLEAM observing strategy, Wayth et al. (2015) estimated the expected thermal noise for GLEAM $30.72 \mathrm{MHz}$ band mosaics for a single fiducial system temperature $T_{\mathrm{f}}$. Table 1 shows their thermal noise estimates for a pointing at declination $-26.7 \mathrm{deg}$ assuming $T_{\mathrm{f}}=200 \mathrm{~K}$. Our thermal noise estimates for GLEAM 7.68 MHz subband mosaics are shown in Fig. 6; they were obtained by multiplying the values in Table 1 by $\sqrt{\frac{30.72 \mathrm{MHz}}{7.68 \mathrm{MHz}} \frac{T_{\mathrm{sys}}+T_{\mathrm{rec}}}{T_{\mathrm{f}}}}=2 \frac{T_{\mathrm{sys}}+T_{\mathrm{rec}}}{T_{\mathrm{f}}}$, where $T_{\text {sys }}$ is the system temperature at the central frequency of the subband. 


\begin{tabular}{cc}
\hline $\begin{array}{c}\text { Frequency } \\
(\mathrm{MHz})\end{array}$ & $\begin{array}{c}\text { Thermal noise sensitivity } \\
(\mathrm{mJy} / \text { beam })\end{array}$ \\
\hline 87.7 & 1.7 \\
118.4 & 1.9 \\
154.2 & 2.1 \\
185.0 & 2.2 \\
215.7 & 2.4 \\
\hline
\end{tabular}

Table 1: GLEAM expected thermal noise sensitivity at a declination of $-26.7 \mathrm{deg}$, assuming a bandwidth of $30.72 \mathrm{MHz}$ and $T_{\mathrm{f}}=200 \mathrm{~K}$, as indicated by Wayth et al. (2015).

\subsection{Classical confusion}

When the density of faint extragalactic sources becomes too high for them to be clearly resolved by the array, the deflections in the image will include the sum of all the unresolved sources in the main lobe of the synthesised beam. This effect is known as classical confusion and only depends on the source counts and the synthesised beam area (Condon, 1974).

We use the method of probability of deflection, or $P(D)$ analysis (Scheuer, 1957), to quantify the classical confusion, $\sigma_{\mathrm{c}}$, in GLEAM. The deflection $D$ at any pixel in the image is the intensity in units of mJy/beam. Given a source count model and synthesised beam size, we use Monte Carlo simulations to derive the exact shape of $P_{\mathrm{c}}(D)$, the $P(D)$ distribution from all sources present in the image, in each subband. We then estimate the rms classical confusion noise from the core width of this distribution.

In the flux density range 6-400 mJy, the Euclidean normalised differential counts at $153 \mathrm{MHz}$ from Williams, Intema \& Röttgering (2013), Intema et al. (2011) and Ghosh et al. (2012) are well represented by a power law of the form $\frac{d N}{d S}=k S^{-\gamma} \mathrm{Jy}^{-1} \mathrm{sr}^{-1}$, with $k=6998$ and $\gamma=1.54$ (see Fig. 2). The $153 \mathrm{MHz}$ differential source counts continue to decline at $S_{153} \lesssim 10 \mathrm{mJy}$ and no flattening of the differential count at low flux densities (e.g. as seen at $1.4 \mathrm{GHz}$ ) has been detected. We use two source count models in the Monte Carlo simulations to derive $P_{\mathrm{c}}(D)$. The counts are modelled as

$$
n(S) \equiv \frac{\mathrm{d} N}{\mathrm{~d} S} \approx\left\{\begin{array}{l}
k_{1}\left(\frac{S}{\mathrm{Jy}}\right)^{-\gamma_{1}} \mathrm{Jy}^{-1} \mathrm{sr}^{-1} \text { for } S_{\text {low }} \leq S<S_{\mathrm{mid}} \\
k_{2}\left(\frac{S}{\mathrm{Jy}}\right)^{-\gamma_{2}} \mathrm{Jy}^{-1} \mathrm{sr}^{-1} \text { for } S_{\mathrm{mid}} \leq S \leq S_{\mathrm{high}}
\end{array}\right.
$$

The values of the source count parameters $k_{1}, \gamma_{1}, k_{2}, \gamma_{2}, S_{\text {low }}, S_{\text {mid }}$ and $S_{\text {high }}$ for each model are provided in Table 2. Model A corresponds to the case where there is no flattening in the counts below $6 \mathrm{mJy}$. In model $\mathrm{B}$, the source count slope is set to 2.2 below $6 \mathrm{mJy}$; the purpose of model $\mathrm{B}$ is to explore how sensitive the classical confusion noise is to a flattening in the source count slope below $6 \mathrm{mJy}$.

We extrapolate the two $153 \mathrm{MHz}$ source count models to the central frequency of each GLEAM subband, assuming each source varies with frequency as $S \propto v^{\alpha}$, by applying the method described in Waldram et al. (2007). We assume three different values of $\alpha$ : $-0.5,-0.7$ and -0.9 .

We derive $P_{\mathrm{c}}(D)$ for each GLEAM subband and source count model as follows: we simulate a noise-free image containing point sources at random positions, assigning their flux densities 


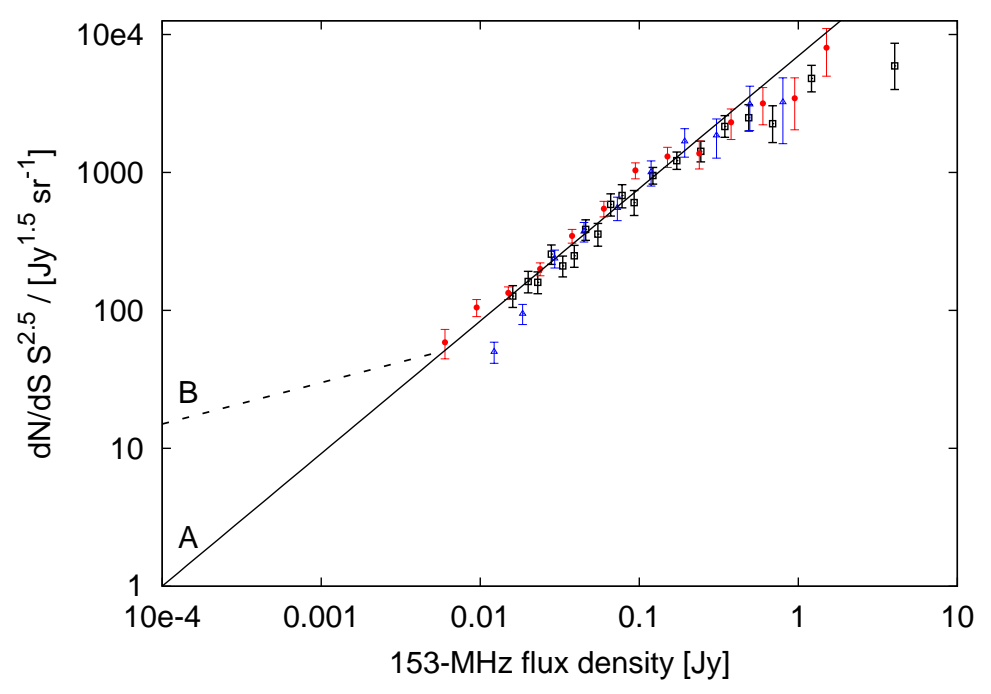

Figure 2: The black squares, red circles and blue triangles show the Euclidean normalised differential counts at $153 \mathrm{MHz}$ from Williams, Intema \& Röttgering (2013), Intema et al. (2011) and Ghosh et al. (2012), respectively. The solid and dashed lines show source count models A and B, respectively.

\begin{tabular}{rrrrrrrr}
\hline Model & $k_{1}$ & $\gamma_{1}$ & $k_{2}$ & $\gamma_{2}$ & $\begin{array}{r}S_{\text {low }} \\
(\mathrm{mJy})\end{array}$ & $\begin{array}{r}S_{\text {mid }} \\
(\mathrm{mJy})\end{array}$ & $\begin{array}{r}S_{\text {high }} \\
(\mathrm{mJy})\end{array}$ \\
\hline A & 6998 & 1.54 & 6998 & 1.54 & 0.1 & 6.0 & 400 \\
B & 237.9 & 2.200 & 6998 & 1.54 & 0.1 & 6.0 & 400 \\
\hline
\end{tabular}

Table 2: Parameter values adopted to model the $153 \mathrm{MHz}$ counts.

according to the source count model. Sources with flux densities ranging between 0.1 and $400 \mathrm{mJy}$ are injected into the image using the MIRIAD task IMGEN (Sault, Teuben, \& Wright, 1995). The simulated point sources are convolved with the Gaussian restoring beam of the subband image; we do not attempt to model the sidelobe confusion. We obtain $P_{\mathrm{c}}(D)$ from the distribution of pixel values in the simulated image. The width of the distribution is measured by dividing the interquartile range by 1.349 , i.e. the rms for a Gaussian distribution. Some examples of $P_{\mathrm{c}}(D)$ are shown in Fig. 3.

Fig. 4 shows that models A and B diverge at higher frequency, indicating that sources below $6 \mathrm{mJy}$ are too faint to contribute significantly to the confusion noise except at the highest frequencies where the beam size is smallest.

\subsection{Sidelobe confusion}

Additional noise is introduced into an image from the combined sidelobes of undeconvolved sources, i.e. from the array response to sources below the source subtraction cut-off limit and to sources outside the imaged FoV. This effect is known as sidelobe confusion. Since the MWA has many baselines and no regularity in the aperture, sidelobes from any single short observation have a nearly random distribution in the image and are not easily distinguishable from other noise terms. 


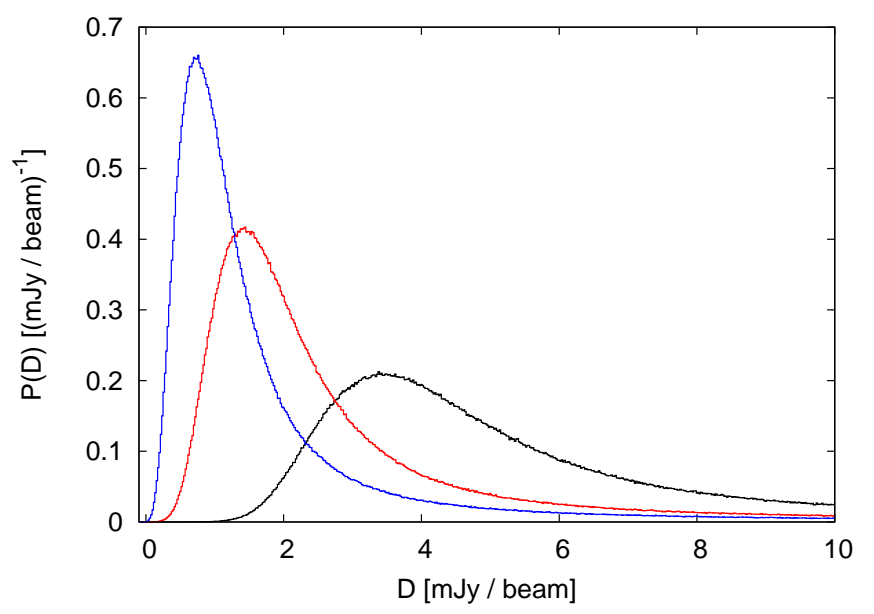

Figure 3: $P_{\mathrm{c}}(D)$ distributions at $122 \mathrm{MHz}$ (black), $158 \mathrm{MHz}$ (red) and $189 \mathrm{MHz}$ (blue) corresponding to source count model B and $\alpha=-0.7$.

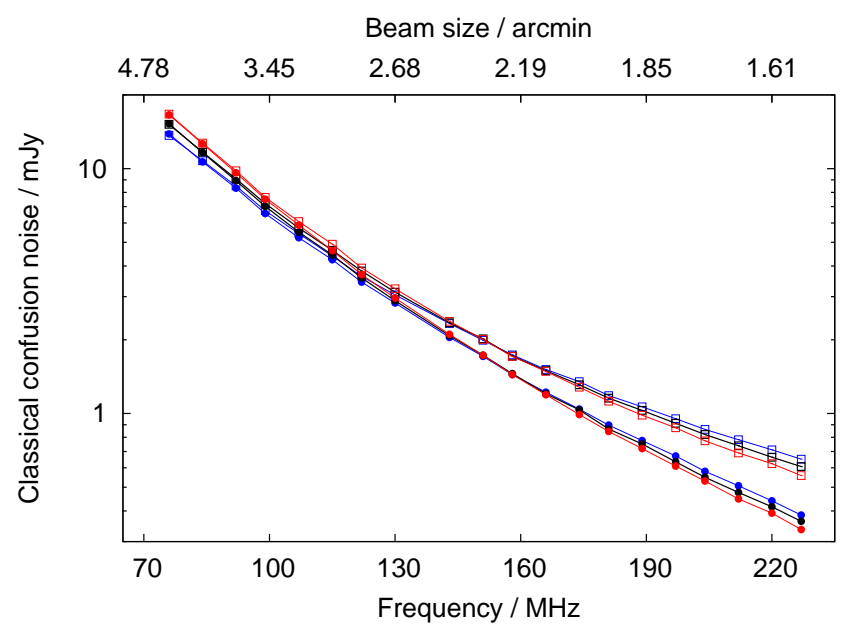

Figure 4: The circles and squares show estimates of $\sigma_{\mathrm{c}}$ using source count models A and B, respectively. These source count models are extrapolated to the GLEAM subband frequencies (72$231 \mathrm{MHz}$ ) assuming $\alpha=-0.5$ (blue), $\alpha=-0.7$ (black) and $\alpha=-0.9$ (red).

In order to draw conclusions about the degree of sidelobe confusion, we compare our estimates of the system noise and classical confusion noise with the measured rms noise in one of the deepest regions of GLEAM.

We measure the mean rms noise in a region within 8.5 deg of the Chandra Deep Field-South (CDFS) at $\mathrm{J} 2000 \alpha=03^{\mathrm{h}} 30^{\mathrm{m}} 00^{\mathrm{s}}, \delta=-28^{\circ} 00^{\prime} 00^{\prime \prime}$. This region lies close to zenith (i.e. at $\delta=-26.7 \mathrm{deg}$ ) and $55 \mathrm{deg}$ from the Galactic Plane. As an example, Fig. 5 shows the lowest GLEAM subband image of this region of sky. We use BANE ${ }^{1}$ to calculate a noise image from the interquartile range of pixels in regions of size $20 \times 20$ synthesised beams.

Fig. 6 reveals that the thermal and classical confusion noise are much lower than the measured

${ }^{1}$ https://github.com/PaulHancock/Aegean 


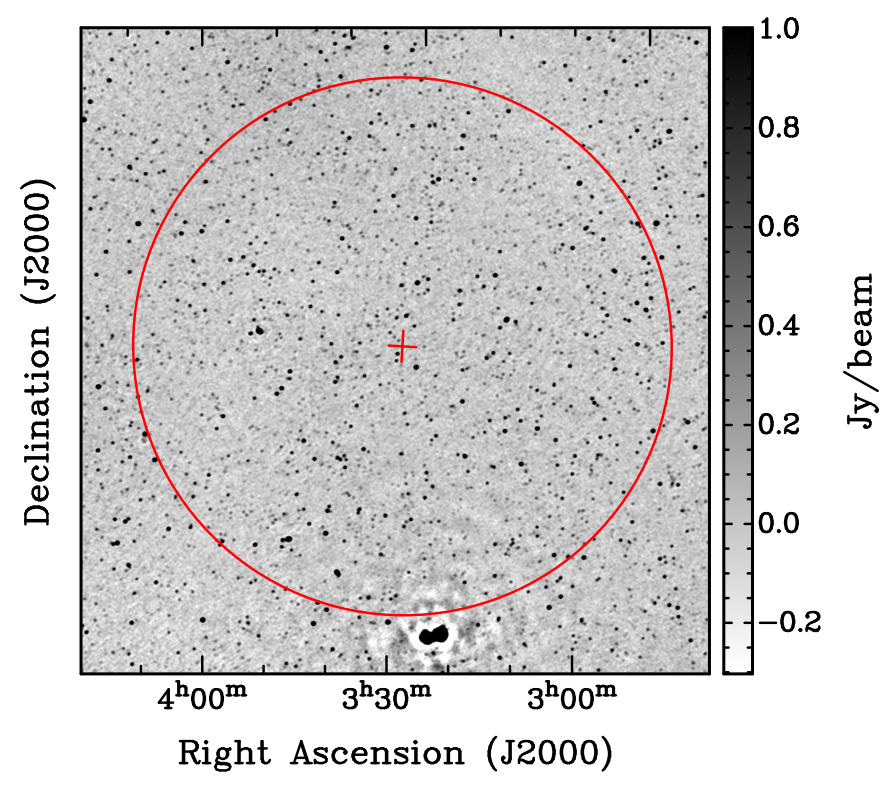

Figure 5: Image of a section of the lowest GLEAM subband image at 72-80 MHz centred on CDFS. The region in which the mean rms noise is measured is shown bounded in red.

noise at all frequencies. From this, we conclude that the background noise is primarily due to sidelobe confusion. Possible origins of the sidelobe confusion are the limited CLEANing depth, far-field sources that have not been deconvolved, and residuals of ionospheric smearing.

\section{Conclusions and future work}

Our initial work suggests that the background noise in GLEAM images is primarily due to sidelobe confusion. This is a consequence of the large FoV and the huge number of detected sources. A similar result was found by Franzen et al. (2016) in one of the deepest images ever made with the MWA, a single MWA pointing image of an Epoch of Reionisation field at $154 \mathrm{MHz}$ with a resolution of $2.3 \mathrm{arcmin}$ (Offringa et al., 2016). This image is affected by sidelobe confusion noise at the $\approx 3.5 \mathrm{mJy} / \mathrm{beam}$ level, and the classical confusion limit is $\approx 1.7 \mathrm{mJy} / \mathrm{beam}$.

In future work, we will determine the noise contribution in different regions of GLEAM (e.g. close to the Galactic plane, powerful radio sources etc.), and investigate how these vary across 72-231 MHz and where best improvements may be made.

\section{Acknowledgments}

This scientific work makes use of the Murchison Radio-astronomy Observatory, operated by CSIRO. We acknowledge the Wajarri Yamatji people as the traditional owners of the Observatory site. CAJ thanks the Department of Science, Office of Premier \& Cabinet, WA for their support through the Western Australian Fellowship Program. Support for the operation of the MWA is provided by the Australian Government Department of Industry and Science and Department 


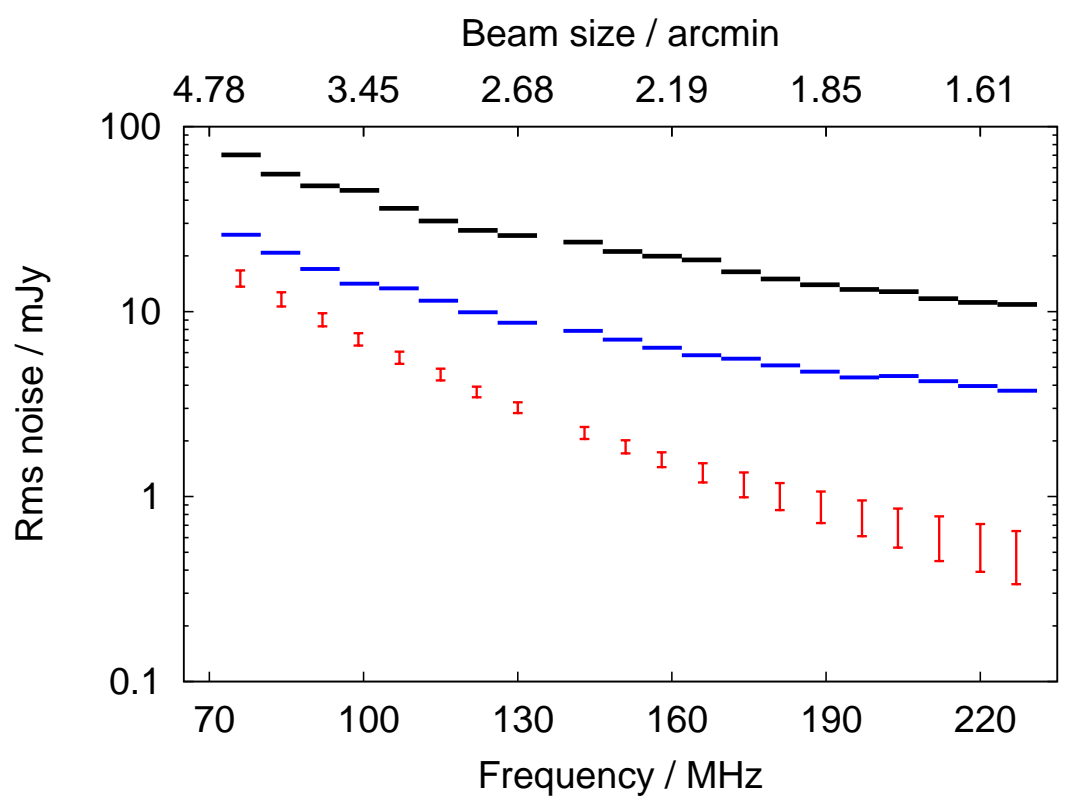

Figure 6: Black horizontal bars: mean rms noise in the $7.68 \mathrm{MHz}$ subband images in a region within $8.5 \mathrm{deg}$ from CDFS. The approximate beam size is shown on the top. Blue horizontal bars: expected thermal noise sensitivity (Wayth et al., 2015). Red points: range of classical confusion noise estimates.

of Education (National Collaborative Research Infrastructure Strategy: NCRIS), under a contract to Curtin University administered by Astronomy Australia Limited. We acknowledge the iVEC Petabyte Data Store and the Initiative in Innovative Computing and the CUDA Center for Excellence sponsored by NVIDIA at Harvard University.

\section{References}

Condon J., 1974, ApJ, 188, 279

Dewdney P. et al., 2010, SKAMemo \#130, SKA Phase 1: Preliminary System Description, www.skatelescope.org/publications/

Franzen T. M. O. et al., 2016, MNRAS, submitted

Ghosh A., Prasad J., Bharadwaj S., Ali S. S., Chengalur J. N., 2012, MNRAS, 426, 3295

Intema H. T., van Weeren R. J., Röttgering H. J. A., Lal D. V., 2011, A\&A, 535, A38

Offringa A. R. et al., 2014, MNRAS, 444, 606

Offringa A. R. et al., 2016, MNRAS, accepted

Sault R. J., Teuben P. J., Wright M. C. H., 1995, ASP Conf. Ser., 77, 433

Scheuer P. A. G., 1957, Proceedings of the Cambridge Philosophical Society, 53, 764

Tingay S. J. et al., 2013, PASA, 30, e007

van Haarlem M. P. et al., 2013, A\&A, 556, A2

Waldram E. M., Bolton R. C., Pooley G. G., Riley J. M., 2007, MNRAS, 379, 1442

Wayth R. B. et al., 2015, PASA, 32, e025

Williams W. L., Intema H. T., Röttgering H. J. A., 2013, A\&A, 549, A55 